\title{
Market Power Mitigation in the Colombian Electricity Market through On-Site Generation and Demand Response
}

\author{
Julián A. Rojas, Nicanor Quijano, Ángela Cadena \\ Department of Electrical and Electronic Engineering, Los Andes University, Bogotá, \\ Colombia
}

\begin{abstract}
This article evaluates the active demand-side participation in the Colombian electricity market as a strategy to mitigate the apparently market power. Real energy and price offers from years 2000 to 2014 are used to characterize the supply curves of the model. The model is developed as a potential game where the strategies available are each of the firms (mostly hydro-based) that are supposed to behave strategically. A replicator dynamics approach is used to obtain the Nash equilibrium of the game. The results show that for acceptable levels of demand-side participation the energy price is reduced in approximately 58\% when firms have the opportunity to exercise market power. With an additional model based on time-of-use (TOU) prices it is borne out the fact that an active demand-side participation helps improving the market efficiency reducing the ability to exercise market power, among others.
\end{abstract}

Keywords: Cogeneration, Demand Response, Evolutionary Game Theory, Market Power, Self-Generation.

JEL: C73, L13, L43, L94

*This work has been supported in part by project Silice III Codensa-Colciencias under Grant P12.245422.006 and, also by the program Jóvenes Investigadores 2015, by Colciencias.

Email addresses: ja.rojas941@uniandes.edu.co (Julián A. Rojas), nquijano@uniandes.edu.co (Nicanor Quijano), acadena@uniandes.edu.co (Ángela Cadena) 


\section{Introduction}

Market power is defined as the ability of a firm, or a group of firms, to alter and maintain prices above the levels that would prevail under competition [1]. These actions turn out to be harmful for the market operation because they carry several inefficiencies. For example, higher prices may be seen as a signal for lack of infrastructure leading to oversizing; the demand side would obtain a welfare loss that is commonly transferred to the producers as a gain. Not in vain The Sveriges Riksbank Prize in Economic Sciences in Memory of Alfred Nobel 2014 was awarded to professor Jean Tirole for "his analysis of market power and regulation" [2].

As it is stated in [3], the electricity sector possesses characteristics that make it very susceptible to the exercise of unilateral market power. Demand has to be satisfied all the time of operation because it is too expensive to store the eletricity produced. The good has to be delivered through a transmission network that is commonly congested (geographical profiteering). Large generation facilities are typically owned by few firms (oligopoly characteristic). There is a high degree of uncertainty with the availability of the generation units when needed for the operation. Taking into account the above, it is important to have institutions (regulator, expert committees) pending the behaviors of generation firms.

Nevertheless, it is important to remark that it is totally impossible to prevent firms from exercising unilateral market power. In [4] it is stated that markets function most efficiently when incentives to exercise market power are present. So in most cases, the cure may be worse than the disease. The regulatory actions should anticipate the possible damages caused by the market power rather than formulate new policies.

Trying to identify the presence of strategic behaviors and to measure the market power levels is a challenging task. Straight-forward techniques that assist in getting this calculations, like the Hirschman-Herfindahl Index (HHI) or the Lerner Index, are useful for initial analyses. However, they exhibit some weakness when used in restructured eletricity markets [5]. Exercise of market power in electricity markets is highly related with the fact that many firms could own generation and distribution assets. The vertical integration degree is an issue that cannot be seen by these methods. Furthermore, techniques like HHI assumes that all agents behave in the same oligopolistic manner, nonetheless there are some small firms that behave in a competitive way. 
In an effort to limit these trust behaviors, demand response programs can provide great help. One of the benefits of these programs is precisely their potential to prevent the exercise of market power by electric power producers. Demand response is defined as the changes in electricity usage by end-use customers from their normal consumption patterns in response to changes in the price of electricity over time [6]. In an ideal scenario, the customers would shift some operations from critical peak periods to off-peak periods due to high prices of electricity; and customers could use on-site generation for their supply, that is self-generation or cogeneration (that typical industries obtain from their processes). Generally, demand response programs are classified into incentive-based and price-based [6]. Within the incentive-based ones are the direct control, interruptible programs, demand bidding, capacity market, among others; and belonging to the price-based programs are time of use (TOU), critical peak pricing (CPP) and real time pricing (RTP) [7]. In this work, we focus our attention in the demand bidding program, through the on-site generation, as the mechanism to mitigate market power.

The strategic behavior of producers, which takes place in presence of market power, has been commonly studied from a game theoretic perspective. Authors in [8] have proposed a non-cooperative game when trading energy between energy storage units like electric vehicles and arrays of batteries. They have proven the existence of a Nash equilibrium when the utility function has a discontinuity and have designed a strategy-proof mechanism for the energy trade. Some other biomimicry approaches have taken place. In [9], the authors have used artificial bees colony and cuckoo search algorithms as optimization tools to propose an adaptive technique based modeling of optimal bidding strategies for an electricity market. Authors in [10] have presented a methodology for an optimal bidding strategy of a supplier considering transmission congestion (and so the possible geographical market power). They have solved a bi-level optimization problem using bacteria foraging optimization, where the lower level problem represents market clearing process and, the upper level problem represents the supplier's profit maximization function.

Some contributions have been made in mechanism design theory [11] [12]. The mechanisms are such that are individually rational, budget balanced, price efficient and, guarantees the existence of a Nash equilibrium corresponding to the maximization of the sum of producers' and consumers' utilities. In [13], the authors have built a model to simulate a hydrothermal electric power market based on simple bids; using dynamic programming, a Nash- 
Cournot equilibrium is determined to analyze the consequences of different strategies to be adopted by generators. Properly, studies have been made for the Colombian electricity market. In [14], it is concluded that since 2009, when regulatory changes took place in the Colombian electricity market, the producers exercise of market power have increased. Consumer surplus has decreased and, if efficiency has increased, then these gains have not been transferred to the consumers. These conclusions are tied to the correct calculation of plants' marginal costs, although the results are demonstrated to be robust. In [5], a dynamic Cournot model is proposed to evaluate the possible exercises of market power in the electricity environment. This technique is proposed, instead of indexes stated above, taking advantage of the large amount of data that is available within an electricity market. In [15], a market power analysis for the Colombian electricity market is performed using the technique proposed above. A dynamic Cournot model is used to evaluate the impacts of possible mergers.

The main contributions of this article are: 1) the modeling of the dynamic Nash-Cournot interactions within a potential game framework; 2) the use of population dynamics, like the replicator ones, to obtain a Nash equilibrium for the model; and, 3) the analysis of market power behaviors when a demand response program is present. The remainder of the article is organized as follows. Section 2 states the parameters and equations that we use in the model. It is also presented the periods we are using for the model with an emphasis on climate conditions. Section 3 explains the dynamics we have used to solve the proposed game. Section 4 shows the results obtained with the active demand-side participation in the market. In Section 5 we present some discussions about the results obtained with the model. Finally, we present the concluding remarks in Section 6 .

\section{The Model}

\subsection{Colombian Electric Industry}

We begin by describing the electricity sector in Colombia. Electricity market is a predominantly hydro-based one. Of a total net effective installed capacity of $15489 \mathrm{MW}^{1}, 10315 \mathrm{MW}$ correspond to hydro sources, $4402 \mathrm{MW}$ to thermal sources, 694.7 MW to minor plants (plants which effective capacity do not exceed $20 \mathrm{MW}$ ) and, 77.3 MW to cogenerator sources [17].

\footnotetext{
${ }^{1}$ Values for end of 2014
} 
Roughly speaking, it accounts for a $70 \%$ of hydro plants and a $30 \%$ for thermal plants. The energy demand for year 2014 was $63571 \mathrm{GWh}$. Manufacturing industries represent a $45.5 \%$ of this value and mining sector a $21 \%$, the rest is represented by social services, trade sector, public services, transport, among others.

Generation sector in Colombia has an oligopolistic characteristic. While there are 56 registered generators, only 5 large firms own the $80 \%$ of the effective installed capacity. As seen in Table 1, these five large firms have in common their hydro-based source. This characteristic makes the Colombian electricity market very susceptible to the exercise of market power. In presence of collusion, tacit or explicit, any of the 5 large firms may decide to withhold water in their reservoirs and not offer energy to the market. This action would make a raise in the spot price bringing additional benefits to the firm.

Table 1: Effective installed capacity by firms in Colombia.

\begin{tabular}{lcllll}
\hline \multirow{2}{*}{ Firm } & \multirow{2}{*}{ Capacity [MW] } & \multicolumn{5}{c}{ Technology } \\
\cline { 3 - 6 } & & Hydro & Thermal & Wind & Cogeneration \\
\hline EPM & 3550 & 2990 & 491 & 19 & 5 \\
EMGESA & 3011.8 & 2600 & 412 & & \\
ISAGEN & 3001 & 2706 & 276 & 52 \\
CELSIA & 1777.7 & 1019 & 770 & & \\
AES CHIVOR & 1000 & 1000 & & & \\
OTHERS & 3148.5 & 450 & 2720 & &
\end{tabular}

\subsection{Dynamic Cournot Model}

We have used the model proposed in [5] and specifically in [15] as a base for the present work. We have assumed that the five largest firms conform an oligopolic group and behave strategically. The rest of the firms have been assumed to behave competitively. Assuming a rational behavior, the objective of each one of the strategic firms can be expressed as the solution 
of the following maximization problem:

$$
\begin{array}{ll}
\underset{q_{i t}}{\operatorname{maximize}} & \sum_{t}\left(p_{t}\left(Q_{t}^{R}\right) q_{i t}-c q_{i t}-g\left(p_{t}\left(Q_{t}^{R}\right)-K\right)\right) \\
\text { subject to } & s_{i t}=s_{i t-1}+f_{i t}-q_{i t} h_{i} \leq M_{i t} \\
& s_{i t}=s_{i t-1}+f_{i t}-q_{i t} h_{i} \geq m_{i t}
\end{array}
$$

where $q_{i t}$ is the quantity of energy generated by $i^{\text {th }}$ firm in period $t$; $c$ is the marginal cost of generating energy; $s_{i t}$ accounts for the level of the reservoir of $i^{\text {th }}$ firm at period $t$, which equals the level of previous period $s_{i t-1}$ plus water intake $f_{i t}$ minus the water used for previous generation; and $h_{i}$ represents an energy to water conversion factor. This level must always be above the minimum values $m_{i t}$ (stated by the Energy and Gas Regulation Commission (CREG), the regulatory institution in Colombia) and obviously below the maximum values $M_{i t}$ given by the physical conditions of the reservoir. In order to explain the other variables, let us make the following definitions.

Definition 2.1. We define $Q_{t}^{R}$ as the residual demand that has to be supplied by the oligopolic group, and is given by,

$$
Q_{t}^{R}=D_{t}-S_{t}(p)
$$

where $D_{t}$ is the demand of period $t$ (assumed inelastic) and $S_{t}(p)$ is the supply curve, of period $t$, of the competitive firms.

Assumption 1. We assume that function $S_{t}\left(p_{t}\right)$ takes the form given by,

$$
S_{t}\left(p_{t}\right)=\frac{1}{c} \sinh ^{-1}\left(\frac{p_{t}-a}{b}\right)-\frac{d}{c},
$$

where variable $p_{t}$ represents the energy price and $a, b, c$, and $d$ are fit coefficients.

Remark 1. Note that one can obtain an inverse demand function by simply solving for $p$ in the definition of $Q_{t}^{R}$, which gives,

$$
p_{t}\left(Q_{t}^{R}\right)=a+b \sinh \left(c\left(D_{t}-Q_{t}^{R}\right)+d\right) .
$$

Remark 2. Also note that the last term in the objective function stands for the bilateral contracts that each firm is able to sign. According to this, firm is selling $g$ units of energy at a $K$ price. 


\subsubsection{Competitive Supply Curves}

The calibration of these $S_{t}(p)$ curves turn out to be important for the model because they express the price at which the energy is going to be paid to the strategic players. We have considered all the hourly bids that were submitted in the Colombian electricity market for the period from year 2000 to 2014. With the aim of constructing the competitive supply curves, we have first excluded the oligopolic group bids from the database. When we only have the competitive bids, we organize them by order of merit and finally we obtain the supply curve. For the sake of precision with the model, we have divided the whole period by its climatic characteristics. In Colombia is very common to come upon with weather phenomenons like El Niño Southern Oscillation (ENSO). It is represented with anomalously warming or cooling temperatures for long periods of time. These situations impact significantly the hydro resources and so the electricity market behavior. Normally, these effects can be seen in the spot price levels. We have collected the spot national price for the fourteen years and plotted in Figure 1. As it is shown, during the last fourteen years two milestones have ocurred. The ENSO occurred in 2010, characterized by an increase in temperatures; and the recently ENSO alert that began in 2012. Keeping in mind the above, we have obtained three different supply curves for the three different periods.

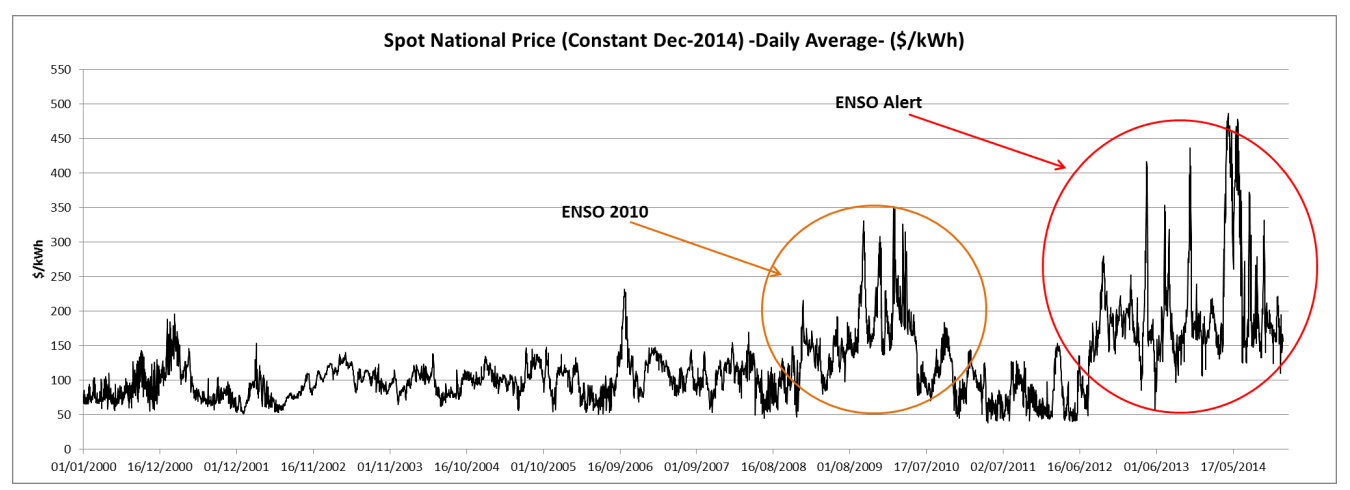

Figure 1: Increases in price for periods between August-2008 to August-2010 and, July2012 to December-2014.

We have also divided each year in dry (January to May) and wet (June to December) months as usual. In Figure 2 to 4 we plot the different approximate supply curves for a range of energy from 0 to $4000 \mathrm{GWh}-$ month. We have shown the graphics in the same range to observe the difference between 


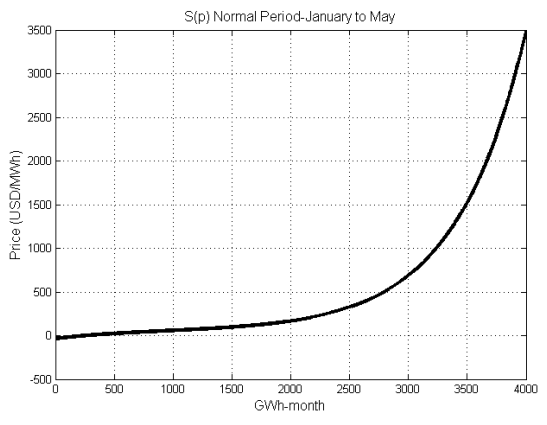

(a) January-May

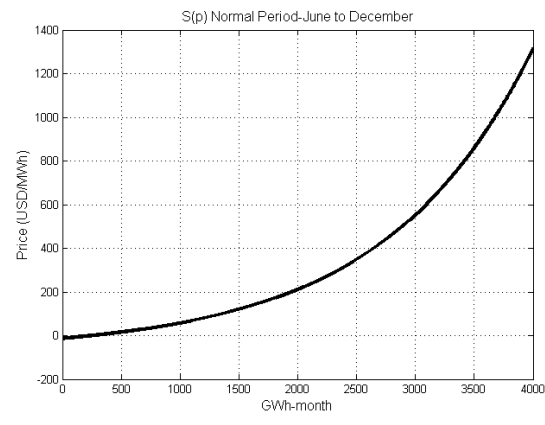

(b) June-December

Figure 2: Supply curves for a normal period of operation are characterized by gradual slopes.

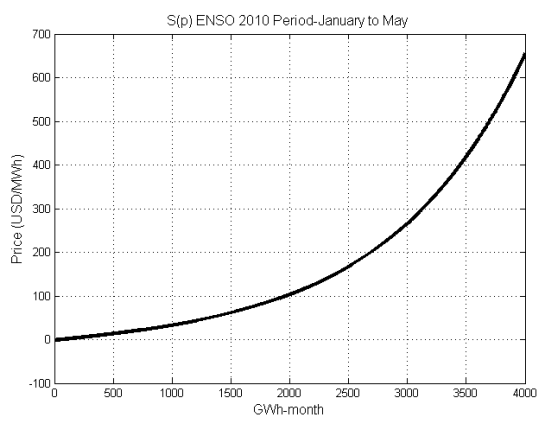

(a) January-May

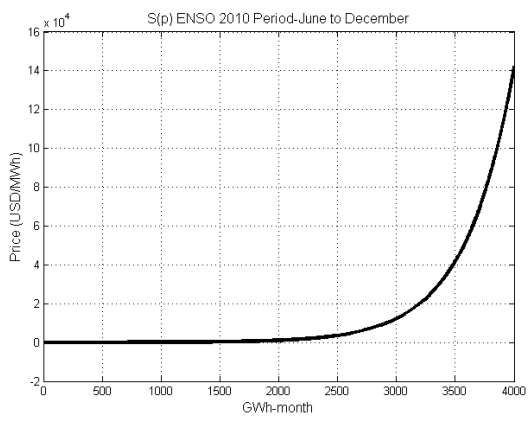

(b) June-December

Figure 3: Supply curves for the ENSO 2010 period show the steep slope for the second half of year.

the prices as the energy generated increases. One interesting fact is the steep slope that exhibit the supply curves for the ENSO (Alert) periods. In Figure $3 \mathrm{~b}$ and Figure $4 \mathrm{~b}$ it can be observed that the energy reach price levels of $10^{4}$ order. Moreover, for the period of alert in 2012 from January to May (see Figure 4a), the prices rise at levels of $10^{6}$ order. Definitely, this would be the prices if there had been any regulatory intervention. Notwithstanding, the regulator is very intesive when these phenomenons are present, avoiding any unpleasant surprise. 


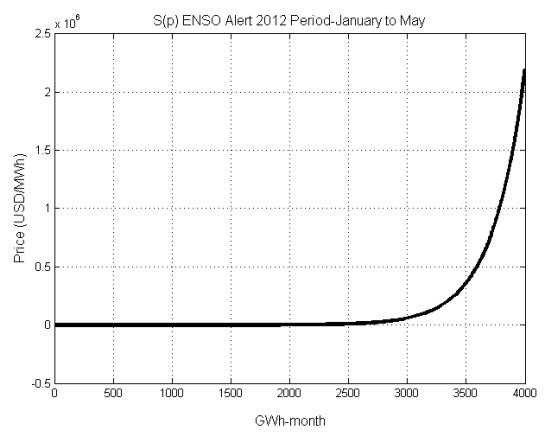

(a) January-May

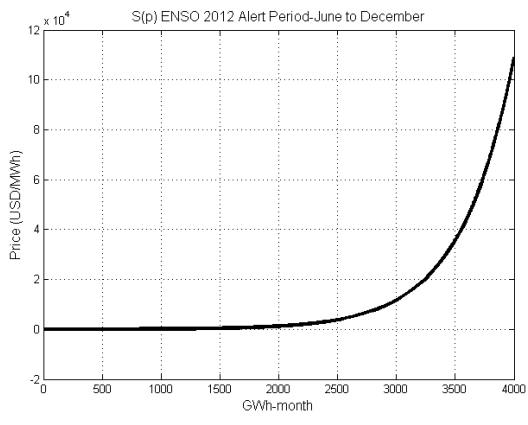

(b) June-December

Figure 4: Supply curves for the alert of ENSO 2012 period show exceptional slopes for both halfs of year.

\subsubsection{Other parameters}

We have provided the model with some other parameters. The values of demand act as a driver of the model. These values have been taken from the document Projection of Demand for Electricity and Maximum Power in Colombia [18] prepared by the Mining And Energy Planning Unit (UPME). They have used an econometric model that allows to reflect the correlations between increases in demand and the electricity production or, some macroecnomic index like GDP. Furthermore, they perfom periodic reviews to correct the predicted values and obtain more accurate results. The document gives values until year 2029 for three scenarios: high, medium, and low. Some sensitivity analysis has been made with the values.

We have also taken into account information about the rivers that provide their flow to the different reservoirs that serve the national grid. Specifically we have collected the energy average value of the rivers (in GWh) from the market operator. These values have been used as the water intakes for the firms. Finally, we have requested the minimun and maximum operating level for each reservoir; these are going to be our constraints for the model.

\subsection{Self-generation and Cogeneration}

Cogeneration or Combined Heat and Power (CHP) is defined as the simultaneous generation of useful heat and power from a single fuel or energy source, close to the point of use [20]. Self-generator, meanwhile, is an agent that consumes all the energy that produces; it only uses the national grid as a backup. In Colombia, initially Government Laws 142 and 143 of 1994, 
began to talk about these type of agents. Nowadays, the recently enacted Law 1715 of 2014 reorganized the concepts around renewable energies and established the guidelines that must be taken towards these forms of generation. One can set forth some benefits of these sorts of generation. Replacing fossil fuels by biomass, as bagasse, brings environmental improvements like a GHG emissions reduction; which is the case of the sugar mills in Colombia. For the cogenerators, an economic incentive (taxes reduction) can also be achieved. Lastly, and the one that is concerned within this work, these on-site generation is an important demand-side form of participation that can help reducing the market power of the largest firms.

The authors in [19] have aimed to inventory the self and cogeneration capacities installed and the possible projections for the next years in Colombia. It is a very thorough study that firstly defines what exactly sectors were supposed to be quantified. Then the information is collected through specials forms that were designed by the authors. The information is classified by plant size, energy intensity, thermal floor and, production line (in the case of petroleum sector). The final results are shown in Table 2 . It can be seen that the petroleum sector leads the field of self-generation with an $80 \%$ of participation, followed by the industry sector that accounts for a $19.6 \%$. On the other hand, the industry sector leads the cogeneration capacity installed with a $79.8 \%$ of participation, while petroleum sector stays with a $20.2 \%$.

As stated before, the study has tried to estimate the projections of the potentials found in the survey. The projections are made using an econometric model that considers the most important variables which may have an impact on the generation types. The values obtained are presented in Table 3. It is important to remark the huge potential that provides sugar and oil palm sector. They provide almost half of the projections of each year. The study has been very careful in estimating these projections due to the rising interest in these type of technologies.

Table 2: Inventory of Self-Generation and Cogeneration in Colombia [19].

\begin{tabular}{lccc}
\hline Sector & $\begin{array}{c}\text { Self-generation } \\
{[\mathbf{M W}]}\end{array}$ & $\begin{array}{c}\text { Cogeneration } \\
{[\mathbf{M W}]}\end{array}$ & $\begin{array}{c}\text { Total } \\
{[\mathbf{M W}]}\end{array}$ \\
\hline Industry & 234 & 596.7 & 830.7 \\
Petroleum & 955 & 151 & 1050 \\
Commercial/Public & 4.1 & 0 & 4.1 \\
TOTAL & 1193.1 & 747.7 & 1940.8
\end{tabular}


Table 3: Projections of Self-Generation and Cogeneration potentials [19].

\begin{tabular}{lccccc}
\hline & \multicolumn{5}{c}{ Capacity [MW] } \\
\hline Industry & 2015 & 2016 & 2017 & 2018 & 2019 \\
Coal Self-Generation & 67.2 & 72.1 & 77.3 & 82.9 & 88.9 \\
Coal Cogeneration & 29 & 31.2 & 33.5 & 36 & 38.7 \\
Gas Cogeneration & 16.6 & 15.9 & 15.2 & 14.6 & 13.9 \\
Total & $\mathbf{1 1 2 . 8}$ & $\mathbf{1 1 9 . 2}$ & $\mathbf{1 2 6}$ & $\mathbf{1 3 3 . 5}$ & $\mathbf{1 4 1 . 5}$ \\
$\begin{array}{l}\text { Petroleum } \\
\text { Self-Generation }\end{array}$ & 63.6 & 139.4 & 24 & 724 & 24 \\
Cogeneration & 112.8 & 59 & & & \\
Total & $\mathbf{1 7 6 . 4}$ & $\mathbf{1 9 8 . 4}$ & $\mathbf{2 4}$ & $\mathbf{7 2 4}$ & $\mathbf{2 4}$ \\
$\begin{array}{l}\text { Sugar Sector } \\
\text { Cogeneration }\end{array}$ & 260 & 290 & 360 & & \\
Oil Palm Sector & & & & & \\
Cogeneration & 246.6 & 258.9 & 271.3 & 283.6 & 295.9 \\
TOTAL & $\mathbf{7 9 5 . 8}$ & $\mathbf{8 6 6 . 5}$ & $\mathbf{7 8 1 . 3}$ & $\mathbf{1 1 4 1 . 1}$ & $\mathbf{2 9 5 . 9}$
\end{tabular}

We have introduced into the model the numbers listed above for both installed capacity and projections as an active demand-side participation. This scenario makes the values of demand reduce significantly. A total installed capacity of 1940 MW (approximately $12.5 \%$ of the net capacity installed) is like having another agent with generation capacity as CELSIA (see Table 1). Evidently the generation is not going to be at maximum capacities because these are not firms whose principal activity is the energy trading. However, some sensitivity analysis has been done to observe the different implications of more or less self and cogeneration.

\subsection{TOU Model}

We have used the model proposed in [24] and present it here before applying it to the Colombian electricity market. The model is based on the principle of marginal cost pricing and consists of a supply, and a demand side.

The objective of the supply side is given by the solution of the following 
minimization problem:

$$
\begin{array}{ll}
\underset{z_{i j h}^{(t)}}{\operatorname{minimize}} & \sum_{t=1}^{T}\left(\sum_{i=1}^{m} \sum_{j=1}^{n} \sum_{h=1}^{H_{j}^{(t)}} r^{t} c_{i}^{(t)} z_{i j h}^{(t)}\right) \\
\text { subject to } & \sum_{i=1}^{n} z_{i j h}^{(t)} \geq d_{j h}^{(t)} \\
& -z_{i j h}^{(t)} \geq-K_{i}^{(t)} \\
& z_{i j h}^{(t)} \geq 0,
\end{array}
$$

where $m$ is the group of generation facilities (indexed by $i$ ), $n$ are the demand blocks (indexed by $j$ ), $T$ are the months of analysis (indexed by $t$ ), $H_{j}^{(t)}$ are the hours in demand block $j$ (indexed by $h$ ), $r$ is the discount factor, $c_{i}^{(t)}$ is the marginal cost of each facility for the month $t, d_{j h}^{(t)}$ is the demand that has to be satisfied for hour $h$ and block $j$ of month $t$, and $K_{i}^{t}$ is the capacity of facility $i$ in month $t$. The decision variable $z_{i j h}^{(t)}$ represents the energy that is provided by firm $i$ in hour $h$ of block $j$ for the month $t$.

Remark 3. Note that the first constraint in (5) deals with the clearing of the market, i.e., the total demand has to be provided by the suppliers; meanwhile, the second and third constraint assure that the energy generated will not exceed the facility capacity, and will be non negative.

Let us derive the Karush-Kuhn-Tucker conditions to solve the above problem [25]. Primal feasibility condition is given by the following set

$$
d_{j h}^{(t)}-\sum_{i=1}^{m} z_{i j h}^{(t)} \leq 0 \quad-K_{i}^{(t)}+z_{i j h}^{(t)} \leq 0 \quad-z_{i j h}^{(t)} \leq 0,
$$

where we simply have rearranged the terms of constraints in (5). Dual feasibility condition is given by

$$
-r^{t} c_{i}^{(t)}=-p_{j h}^{(t)}+u_{i j h}^{(t)}-\mu_{3} \quad p_{j h}^{(t)}, u_{i j h}^{(t)}, \mu_{3} \geq 0,
$$

where $p_{j h}^{(t)}, u_{i j h}^{(t)}$, and $\mu_{3}$ are Lagrange multipliers. The first one is interpreted as the discounted marginal cost of increasing the energy demand of the $j$ th 
block for hour $h$ by a unit; and the second one represents the scarcity rent of the system. Finally the complementary slackness condition is given by

$$
p_{j h}^{(t)}\left(d_{j h}^{(t)}-\sum_{i=1}^{m} z_{i j h}^{(t)}\right)=0 \quad u_{i j h}^{(t)}\left(-K_{i}^{(t)}+z_{i j h}^{(t)}\right) \quad \mu_{3}\left(-z_{i j h}^{(t)}\right)=0 .
$$

Definition 2.2. We define the KKT conditions of the supply side problem as a complementarity problem given by

$$
\begin{aligned}
& z_{i j h}^{(t)} \geq 0 \perp r^{t} c_{i}^{(t)}-p_{j h}^{(t)}+u_{i j h}^{(t)} \geq 0 \\
& p_{j h}^{(t)} \geq 0 \perp \sum_{i=1}^{m} z_{i j h}^{(t)}-d_{j h}^{(t)} \geq 0 \\
& u_{i j h}^{(t)} \geq 0 \perp K_{i}^{(t)}-z_{i j h}^{(t)} \geq 0,
\end{aligned}
$$

where $\perp$ symbol indicates that, at the optimal solution, one of the inequalities must be satisfied as an equality, i.e., the product between both sides must equal zero.

Remark 4. Note that Lagrange multiplier $\mu_{3}$ can be omitted by simply changing the equality in (7) by an inequality, i.e., taking $\mu_{3}$ as a slack variable.

Let us now present the model used for the demand side as in [24].

Definition 2.3. We define a single commodity geometric distributed lag demand model given by

$$
d^{(t)}=a^{(t)}\left(p^{(t)}\right)^{b}\left(d^{(t-1)}\right)^{e}
$$

where $d^{(t)}$ is the demand in month $t, a^{(t)}$ is a number serving as the non-price effects, e.g., social issues, $p^{(t)}$ is the price of electricity in month $t, d^{(t-1)}$ is the demand of the previous month, $b$ is the short-run price elasticity and, $e$ is the long-run price elasticity.

Remark 5. Note that taking natural logarithm of both sides of (12) and extending the model to a multi-commodity case (different demand blocks) one can obtain

$$
\ln \left(D_{j}^{(t)}\right)=A_{j}^{(t)}+\sum_{k=1}^{n} B_{j k} \ln \left(P_{k}^{(t)}\right)+E_{j j} \ln \left(D_{j}^{(t-1)}\right),
$$

where $D, A, B, P, E$ are now matrices containing the variables described above. 
Additional to the parameters already expressed, one can obtain an additional one that measures the fraction of total energy demand during block $j$ of month $t$ that occurs during hour $h$. This parameter $\delta_{j h}^{(t)}=\frac{d_{j h}^{(t)}}{d_{j}^{(t)}}$ is supposed to express the several non-price effects of each hour within a demand block, where $d_{j}^{(t)}=\sum_{h=1}^{H_{j}^{(t)}} d_{j h}^{(t)}$.

The supply and demand model are merged into a square system with six variables, $z_{i j h}^{(t)}, p_{j h}^{(t)}, p_{j}^{(t)}, u_{i j h}^{(t)}, d_{j h}^{(t)}, d_{j}^{(t)}$, and six equations: Equations (9), (10), (11), (13), (14), and (15). Equation (15) ensures that the revenue of the supplier is fully satisfied with the value paid by the consumers. Simulations are performed for the system and the results are shown in Section 4.

$$
\begin{gathered}
d_{j h}^{(t)}=\delta_{j h}^{(t)} d_{j}^{(t)} \\
p_{j}^{(t)}=\sum_{h=1}^{H_{j}^{(t)}} \delta_{j h}^{(t)} p_{j h}^{(t)} r^{-t}
\end{gathered}
$$

\section{Evolutionary Dynamics}

In order to derive the potential game framework, using ideas from [21], let us begin with the next definition.

Definition 3.1. The dynamic Cournot model, stated in Section 2.2 is a population game composed by a single population, which forms a continuum of mass $D_{t}$; the choice of strategies from a set $S$, during game play, gives the set of population states given by, $Q_{t}=\left\{q \in \mathbb{R}_{+}: \sum_{i \in S} q_{i t}=D_{t}\right\}$. Payoff functions $f_{i}: Q_{t} \rightarrow \mathbb{R}$ are continuous maps that assigns each social state a payoff. It also exhibits the following properties:

- The number of agents, which are small parts of demand $D_{t}$, are large.

- Individual agents are small. An agent's action has little effect on other agents' payoff.

- Agents interact anonymously. There is no kind of coordination or cooperation among agents. 
Remark 6. At this point it is important to note that the set of strategies available to the population of agents are each one of the firms that provide energy to the market, i.e., $S=\{$ EPM, EMGESA, ISAGEN, CELSIA, AES CHIVOR, and competitive group $\}$. It could be useful to think the strategies (firms) as different habitats where the agents (small quantities of energy) choose to live (be generated by).

With the objective of deriving the payoff functions, let us introduce the notion of full potential games.

Definition 3.2. Let $f: \mathbb{R}_{+} \rightarrow \mathbb{R}$ be a full population game. $f$ is a full potential game if there exists a continuously differentiable function $V: \mathbb{R}_{+} \rightarrow$ $\mathbb{R}$ satisfying,

$$
\nabla V(q)=f(q)
$$

Remark 7. Note that the fact for obtaining a full population game from a population game is extending the domain of the game from the population state to the entire positive orthant.

Assumption 2. We assume the potential function for our model to be given by,

$$
\begin{aligned}
V\left(q_{i}\right) & =p(Q(x)) q_{i}-c q_{i}-g(p(Q(x))-K)+\epsilon_{1} \log \left(\phi_{i}-q_{i}\right) \\
& +\epsilon_{2} \log \left(q_{\text {imax }}-q_{i}\right)+\epsilon_{3} \log \left(\theta_{i}-q_{i}\right)
\end{aligned}
$$

where reservoirs constraints are represented by the logaritmic barrier functions, tuned by variables $\epsilon_{1,2,3}$; in that way, $\phi_{i}, q_{\text {imax }}, \theta_{i}$ represent the minimum reservoir level, the maximum value of generation due to machine constraints and, the maximum reservoir level.

Having obtained the potential function, we can derive the payoff functions, given by,

$$
f_{i}=\frac{\partial V\left(q_{i}\right)}{\partial q_{i}}=p^{\prime}(Q(x))(q-g)+p(Q(x))-\frac{\epsilon_{1}}{\phi_{i}-q_{i}}-\frac{\epsilon_{2}}{q_{i m a x}-q_{i}}-\frac{\epsilon_{3}}{\theta_{i}-q_{i}} .
$$

Now that we have the framework for describing the interactions between the agents, we continue to model the behavior of the agents who are playing the game. This dynamic model in which each agent sporadically changes of strategy is called a revision protocol [21]. For the game explained above, we 
have chosen the family of imitation revision protocols. Under this protocol, an agent chooses an opponent at random and observes its strategy. The agent would change his strategy to the one that is observing with a probability proportional to some welfare value. Specifically, we use the leading example of imitation protocols, the replicator dynamics.

The replicator dynamics have been used as an appropriate mechanism to model the allocation of a population in an environment. The idea behind this concept is that the population, in this case the demand $D_{t}$, after evolutionary processes converge to an equilibrium where all agents achieve the same profit (in this case the indifference of being generated by any firm) [22]. The dynamics of our model are though given by the replicator equation,

$$
\dot{q}_{i}=q_{i}\left(f_{i}(q)-\bar{f}(q)\right)
$$

where $q_{i}$ is the energy to generate, $f_{i}(q)$ the payoff function as in Eq. (18) and, $\bar{f}(q)=\frac{1}{D} q^{\top} f$, the average fitness.

Remark 8. Note that the dynamics reach the equilibrium whenever $f_{i}\left(q^{*}\right)=$ $\bar{f}\left(q^{*}\right)$. As $V\left(q_{i}\right)$ is strictly concave, when solving the dynamics we can obtain the Nash equilibrium of the game, which is a singleton containing the unique maximizer of the potential function [22].

\section{Results}

\subsection{Dynamic Cournot Model}

Based on the model described in Section 3 we have performed several simulations to observe the effect of demand-side participation. The results obtained are then, the solution of many ODEs system, i.e., the replicator dynamics. Using information from the National Oceanic and Atmospheric Administration (NOAA) about the ENSO phenomenon we have assumed that there would be existence of it in years 2016, 2022 and, 2028 [23]. This information is useful to calibrate the supply curves in an appropriate way. To perform an adequate simulation, we have begun by assuring the existence of the exercise of market power. Producers have been provided with additional generation capacity to simulate this issue. Next, we have introduced the self and cogeneration variables in the model. As a sensitivity analysis, we have used three diferent ratios for these types of generation (25, 50 and, 75 percent of the total estimated). 


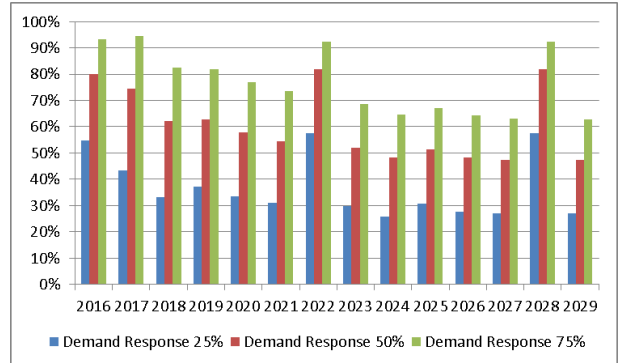

(a) Price reductions when single firm exercise market power.

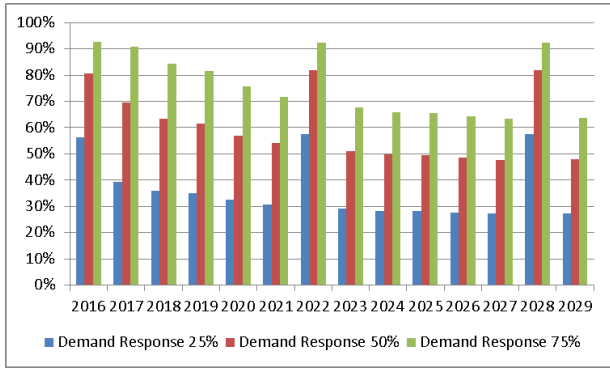

(b) Price reductions when a different firm exercise market power.

Figure 5: Price reductions for single firms exercising market power.

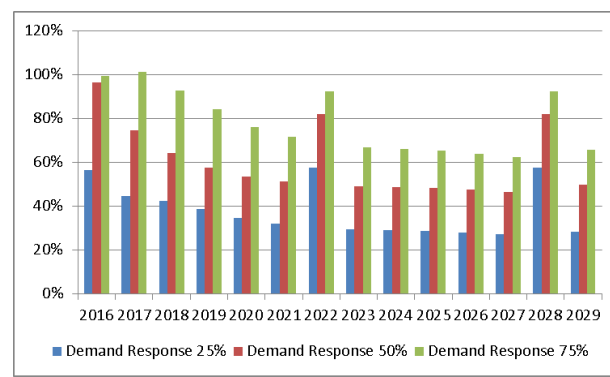

(a) Price reductions when two firms exercise market power.

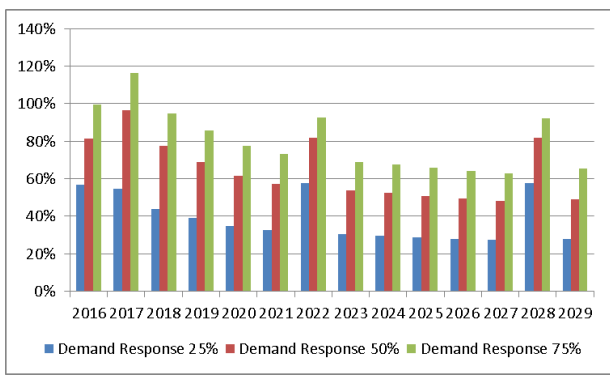

(b) Price reductions when three firm exercise market power.

Figure 6: Price reductions for collusion cases.

For scenarios with the medium projections of demand we have plotted in Figure 5a and 5b the price reductions that are achieved when self and cogeneration are present. The results are very similar, for the case of $25 \%$ we obtain a $37 \%$ reduction, on average; for the case of $50 \%$ and $75 \%$ we obtain $61 \%$ and $77 \%$ reduction on average, respectively. It is important to note the presence of ENSO phenomenons in years 2022 and 2028. We have obtained higher percentages reductions because the prices reached are enormous (recall the statement in Section 2.2.1 about the regulation controls). In Figure 6a and $6 \mathrm{~b}$ we have simulated the price reductions when two and three firms are exercising market power. At first sight the behavior is the same as before. Actually the reductions at this point are greater due to the higher increase in prices after the exercise of market power. We obtain reductions of $39 \%$, $65 \%$, and $80 \%$ for the three scenarios of demand-side participation. There is 
a value in year 2017 that results in a total reduction, i.e., the price becomes negative (issue that makes little sense). The reason for that is the fit that has been made for the supply curves. Trying to perform well with the error, the fit pays more attention to the large values.

\subsection{TOU Model}

In order to make some simulations, we have chosen four months of year 2014 (September, October, November and, December) of operation in the Colombian electricity market. Specifically, hourly demand data is collected. We have divided the day in two demand blocks: on-peak and off-peak period. Each one consisting of twelve hours.

- On-peak period: From hours 8:00 to 19:00

- Off-peak period: From hours 20:00 to 7:00

The values of demand for each block for the four months are shown in Table 4. The values of elasticities have been taken directly from [26] as shown in Table 5. As can be seen, own-elasticities are negative because an increment in the price of a block would lead to less consumption in the same block. On the other hand, cross-elasticities are positive (but not symmetric) because an increase in the price of a demand block would lead to shift consumption to the other block. The value for long-term elasticity is the same for both blocks, i.e., $e_{j j}=0.5$.

Table 4: Demand data for the colombian electricity market

\begin{tabular}{lccc}
\hline$d_{j}^{(t)}[\mathrm{MWh}]$ & on-peak & off-peak & Total \\
\hline August & $2.968 .443,29$ & $2.463 .932,45$ & $\mathbf{5 . 4 3 2 . 3 7 6}$ \\
September & $2.981 .444,07$ & $2.445 .277,18$ & $\mathbf{5 . 4 2 6 . 7 2 1}$ \\
October & $3.040 .361,05$ & $2.480 .971,60$ & $\mathbf{5 . 5 2 1 . 3 3 3}$ \\
November & $2.948 .698,53$ & $2.437 .130,91$ & $\mathbf{5 . 3 8 5 . 8 2 9}$ \\
December & $3.016 .644,28$ & $2.513 .877,31$ & $\mathbf{5 . 5 3 0 . 5 2 2}$ \\
\hline
\end{tabular}

Five technologies have been used for the model: hydro, gas, coal, wind, and self-generation and cogeneration. The marginal costs of each techonology have been taken from UPME data. We have simulated the model in GAMS software using the PATH solver, a special tool for complementarity problems. 
Table 5: Short-run elasticities

\begin{tabular}{lcc}
\hline$b_{j k}$ & on-peak & off-peak \\
\hline on-peak & -0.8 & 0.6 \\
off-peak & 0.5 & -0.7
\end{tabular}

The model returns as an output the TOU prices for each block of demand that would have been instead of the single price. We have simulated the cases with and without self and cogeneration. In Table 6 and 7 the values of demand and TOU prices are shown for the case where no way of demand response is present. As can be seen, the values of demand are higher than the ones that were obtained in Table 4. Essentially, we have obtained an increase of $5 \%$, on average, in demand consumption. This is explained because the prices of energy obtained for the demand blocks are $30 \%$ lower than the single price used above.

Table 6: Demand resulting without self and cogeneration

\begin{tabular}{llll}
\hline$d_{j}^{(t)}[\mathrm{MWh}]$ & on-peak & off-peak & Total \\
\hline September & 3.084 .900 & 2.616 .700 & $\mathbf{5 . 7 0 1 . 6 0 0}$ \\
October & 3.068 .000 & 2.616 .700 & $\mathbf{5 . 6 8 4 . 7 0 0}$ \\
November & 3.061 .400 & 2.641 .300 & $\mathbf{5 . 7 0 2 . 7 0 0}$ \\
December & 3.051 .400 & 2.626 .500 & $\mathbf{5 . 6 7 7 . 9 0 0}$ \\
\hline
\end{tabular}

Table 7: Energy TOU prices without self and cogeneration

\begin{tabular}{lll}
\hline$p_{j}^{(t)}[\mathrm{USD} / \mathrm{MWh}]$ & on-peak & off-peak \\
\hline September & $\$ 142,57$ & $\$ 138,40$ \\
October & $\$ 175,71$ & $\$ 172,61$ \\
November & $\$ 136,84$ & $\$ 134,55$ \\
December & $\$ 155,78$ & $\$ 154,13$ \\
\hline
\end{tabular}

With the purpose of evaluating the benefits of demand-side participation, we have added the self and cogeneration capacities that were estimated as shown in section 2.3 to the model. The results for this case are shown in 
Tables 8 and 9 . It is shown again that there is an increase of about $8 \%$ in demand consumption. The prices of energy are reduced in $20 \%$, enabling the consumers to demand larger amounts of energy. The reduction in prices also cooperates with the aim of reducing the ability to exercise market power from the huge firms.

Table 8: Demand resulting with self and cogeneration

\begin{tabular}{llll}
\hline$d_{j}^{(t)}[\mathrm{MWh}]$ & on-peak & off-peak & Total \\
\hline September & 3.330 .300 & 2.824 .900 & $\mathbf{6 . 1 5 5 . 2 0 0}$ \\
October & 3.312 .200 & 2.824 .900 & $\mathbf{6 . 1 3 7 . 1 0 0}$ \\
November & 3.305 .000 & 2.851 .500 & $\mathbf{6 . 1 5 6 . 5 0 0}$ \\
December & 3.294 .200 & 2.835 .500 & $\mathbf{6 . 1 2 9 . 7 0 0}$ \\
\hline
\end{tabular}

Table 9: Energy TOU prices with self and cogeneration

\begin{tabular}{lll}
\hline$p_{j}^{(t)}[\mathrm{USD} / \mathrm{MWh}]$ & on-peak & off-peak \\
\hline September & $\$ 97,22$ & $\$ 94,38$ \\
October & $\$ 145,10$ & $\$ 142,53$ \\
November & $\$ 113,00$ & $\$ 111,11$ \\
December & $\$ 128,65$ & $\$ 127,27$ \\
\hline
\end{tabular}

\section{Discussion}

The results shown in Section 4 offer an interesting approach for the evaluation of benefits when self and cogeneration take part in an electricity market, specifically in the Colombian electricity market. We have assured the benefits that on-site generation brings to the electricity market. From the simulations performed with the dynamic Cournot model, it is clear that the bilateral contracts, appart from hedging risk, prevent the exercise of market power. In Colombia, the contracting levels have been quite high. This is a very confidential matter and few information is available to the public. From a report made by the Monitoring Committee of the Wholesale Electricity Market we could obtain contracting levels for the oligopolic firms. The firms provide a $70 \%$ on average of their generation to meet the commitments stated in the 
contracts. While performing the exercise of market power in the simulations we reduce the contract levels of firms.

We have represented the model with a single population potential game. For each year of analysis we have solved an independent game that recibed the quantity of energy generated from the previous year. Nevertheless, once the solution is obtained for some year, the simulation goes forward and does not consider a backward process to adjust the outputs. Consequently the values obtained are not the optimum ones. We have compared those results with the ones obtained by classical approaches and considering the relations between periods. The values are quite similar, so the model is a close approach.

Making use of the powerful tool formulation of a complementarity problem, we have modeled the supply and demand side of an electricity market. The TOU model treated in section 2.4 has shown, in a similar way, how

the active demand-side participation contributes to the reduction of prices (limiting the ability to exercise market power). Apart from verifying this issue, the model has allowed us to calculate the prices for each demand block, under a TOU scheme. One could use careful estimated elasticites to predict the TOU prices (ex-ante) more precisely.

\section{Conclusions}

We have evaluated an active demand-side participation in the Colombian electricity market with the aim of reducing the ability of largest firms to exercise market power. A population dynamic approach have been used to model the behaviors of the players. This is a different approach to the traditional optimization methods that have been used in similar works. Our model does not require the players to know explicitly others' generation to solve the maximization problem, hence the 'tatonnement' procedure is not carried out. In our model, all agents play at a single time exploiting advantages for a real-time pricing market, for example. We have calibrated several supply curves for different periods of analysis, related with the environmental conditions. These supply curves have been then used in the model to emule the future posible events. Using estimates from the Energy and Mining Planning Unit (UPME) we have introduced real self-generation and cogeneration values that act as the responsive demand in the model. Finally, reductions of spot prices have been obtained for different generation scenarios where we have modeled the market power for specific group of agents. Combining a supply and demand side model, we have simulated the market equilibrium 
when operating under a TOU program. We have showed again that a proper demand participation could lead to a reduction in energy price. Simulations presented are the most impactant ones, nonetheless one can perform any simulation with different parameters values.

As a future direction we envision a multipopulation game where all the periods under analyses interact back and forward. This would give us the optimum values for the problem. A precisely estimation of demand elasticities for the local environment can also provide high precision to the model and lead to more robust values.

\section{References}

[1] Glossary of Industrial Organisation Economics and Competition Law, compiled by R. S. Khemani and D. M. Shapiro, commissioned by the Directorate for Financial, Fiscal and Enterprise Affairs, OECD, 1993.

[2] "The Prize in Economic Sciences 2014". Nobelprize.org. Nobel Media AB 2014. Web. 7 Jun $2015 . \quad$ http://www.nobelprize.org/nobel_prizes/economicsciences/laureates/2014/

[3] Wolak, Frank A., "Unilateral Market Power in Wholesale Electricity Markets ", CESifo DICE Report 4 (2), 2006, 10-15

[4] Wolak, Frank, Managing Unilateral Market Power in Electricity (August 2005). World Bank Policy Research Working Paper No. 3691.

[5] Severin Borenstein, James Bushnell, Edward Kahn, Steven Stoft, Market power in California electricity markets, Utilities Policy, Volume 5, Issues 34, JulyOctober 1995, Pages 219-236.

[6] M.H. Albadi, E.F. El-Saadany, A summary of demand response in electricity markets, Electric Power Systems Research, Volume 78, Issue 11, November 2008, Pages 1989-1996,.

[7] Pierluigi Siano, Demand response and smart gridsA survey, Renewable and Sustainable Energy Reviews, Volume 30, February 2014, Pages 461478. 
[8] Yunpeng Wang; Saad, W.; Zhu Han; Poor, H.V.; Basar, T., "A GameTheoretic Approach to Energy Trading in the Smart Grid," IEEE Transactions on Smart Grid, vol.5, no.3, pp.1439,1450, May 2014.

[9] Reddy, V.M.S.; Subramanyam, B.; Kalavathi, M.S., "An adaptive technique based modeling of optimal bidding strategies for competitive electricity market," In Proc. of the 2014 Power and Energy Systems Conference: Towards Sustainable Energy, 2014 , vol., no., pp.1,11, 13-15 March 2014.

[10] Jain, A.K.; Srivastava, S.C.; Singh, S.N.; Srivastava, L., "Bacteria Foraging Optimization Based Bidding Strategy Under Transmission Congestion," IEEE Systems Journal, vol.9, no.1, pp.141,151, March 2015.

[11] Rasouli, M.; Teneketzis, D., "Electricity pooling markets with elastic demand: A mechanism design approach," In Proc. of the 2014 52nd Annual Allerton Conference on Communication, Control, and Computing.

[12] Barreto, C.; Mojica-Nava, E.; Quijano, N., "Incentives-Based Mechanism for Efficient Demand Response Programs," submitted to IEEE Transactions on Automatic Control.

[13] Villar, J.; Rudnick, H., "Hydrothermal market simulator using game theory: assessment of market power," IEEE Transactions on Power Systems, vol.18, no.1, pp.91,98, Feb 2003.

[14] An Evaluation of CREG 0512009 Regulatory Intervention in Colombian Electricity Market. Final Report. Luciano de Castro, Shmuel Oren, Alvaro J. Riascos. December 20, 2013.

[15] Alfredo Garcia, Luis E Arbeláez, Market power analysis for the Colombian electricity market, Energy Economics, Volume 24, Issue 3, May 2002, Pages 217-229.

[16] Rassenti, S. J., Smith, V. L., \& Wilson, B. J. (2003). Controlling market power and price spikes in electricity networks: Demand-side bidding. Proceedings of the National Academy of Sciences, 100(5), 2998-3003.

[17] "Descripción y funcionamiento del Sistema Eléctrico Colombiano". XM.com.co. Web. 8 Jun 2015. 
http://informesanuales.xm.com.co/2014/SitePages/operacion/2-6Capacidad-efectiva-neta.aspx

[18] Proyección de la Demanda de Energía Eléctrica y Potencia Máxima en Colombia. March 2015 Review. Unidad de Planeación Minero Energética (UPME).

[19] Capacidad Instalada de Autogeneración y Cogeneración en Sector de Industria, Petróleo, Comercio y Público del País. Informe Final. Consorcio HART-RE. Diciembre 22, 2014.

[20] CHP/DHC Country Scorecards. International Energy Agency. Web. 9 Jun 2015. http://www.iea.org/G8/CHP/profiles.asp

[21] W. H. Sandholm, Population Games and Evolutionary Dynamics (Economic Learning and Social Evolution), 1st ed. The MIT Press, Jan. 2011.

[22] Pantoja, A., \& Quijano, N. (2011). A population dynamics approach for the dispatch of distributed generators. IEEE Transactions on Industrial Electronics, 58(10), 4559-4567.

[23] ENSO: Recent Revolution, Current Status and Predictions. National Oceanic and Atmospheric Administration. NOAA. Web. 10 Jun 2015. http://www.cpc.ncep.noaa.gov/products/analysis_monitoring/lanina/ enso_evolution-status-fcsts-web.pdf

[24] Celebi, E.; Fuller, J.D., "A Model for Efficient Consumer Pricing Schemes in Electricity Markets," IEEE Transactions on Power Systems, vol.22, no.1, pp.60,67, Feb. 2007.

[25] Bazaraa, M. S., Sherali, H. D., \& Shetty, C. M. (2013). Nonlinear programming: theory and algorithms. John Wiley \& Sons.

[26] TOU Pricing. Adaptive and TOU Pricing Schemes for Smart Technology Integration. ORDECSYS. October 2014. 